\title{
The Role of Functional Feed Additives in Modulating Intestinal Health and Integrity
}

\author{
Andreas Kocher $^{\dagger}$ \\ Alltech Biotechnology P/L, 64-70 Nissan Drive Dandenong South, Vic 3175, Australia
}

\begin{abstract}
One of the biggest challenges for the animal feed industry in the coming years will be to meet the growing demand in animal protein in light of increased cost of feed ingredient as well as tougher restrictions on the use of antimicrobial growth promoters imposed by consumers and governments. A key focus area will be to maximise feed efficiency and minimise nutrient waste. It has been widely acknowledged that the composition of the intestinal microflora is closely related to intestinal health and performance of animals. Advanced microbial techniques have shown a close relationship between bacterial communities and their ability to modulate nutrient absorption and processing. In addition it has been recognised that modulating the immune response has significant impact on overall health as well as overall nutrient demand. Molecular techniques are a useful tool to gain an understanding of the impact of dietary interventions including the use of functional feed additives on specific changes in microbial communities or the immune system. Most these techniques however focus on the evaluation of large changes in bacterial compositions and often underestimate or neglect to recognise small changes in microbial diversity or behaviour changes without any measurable immune response. The key to understanding the relationship between specific nutritional intervention and the impact on health and performance lies in a deeper understanding of the impact of these nutrients on the expression of specific genes or specific metabolic pathways. The development of molecular tools as a result of developments in the field of Nutrigenomics has enabled researchers to study the effects of specific nutrients on the whole genome or in other words, the effect of thousands of genes simultaneously, and has opened a completely different avenue for nutritional research.
\end{abstract}

(Key words : intestinal health, broiler performance, nutrigenomics, functional feed additives, mucin)

\section{INTRODUCTION}

In October 1999, the world population reached 6 billion people and is expected to rise to 10 billion within the next 25 years (United Nation, 2011). The key question facing the world is how to provide enough food for such a rapidly growing population?

Forecast analysis has shown that the economic recovery and growth particularly in Asia will increase the demand for animal protein (meat, egg and milk) by more than $25 \%$ by 2020 (Speedy, 2003). Despite severe climate events in major grain growing areas, it has been reported that genetic improvement and an increase in the area of grain production led to record harvest in recent years. In order to fill the growing demand for food and animal protein maximising the amount of animal protein derived from our cereal is becoming a crucial issue.

The biggest increase in animal protein will be in poultry meat and eggs (Roppa, 2007). Despite the fact that poultry is already a very efficient converter of cereal to animal protein, it will be essential to maximize feed efficiency and minimize waste in this area. Poor intestinal health significantly reduces weight gain but also significantly increases feed conversion ratio (van der Klis, 2010). Considering the increasing demand for feed for intensive animal production even a small reduction in FCR will have significant implications on the overall availability of feedstuff for animal production and subsequently the cost of feed. Traditionally, the poultry industry has been using in-feed antibiotics (AGPs) to increase performance and feed efficiency. Despite the widespread use of AGPs and the general acknowledgment that AGPs have a positive impact on growth performance, its actual mode of action is still poorly understood. The most common argument related to the mode of action of AGPs is the impact these compounds have on the intestinal microflora. However the low inclusion level of growth promoting antibiotics, different classes of AGPs with varying spectra, and the significant changes in the microflora during

\footnotetext{
${ }^{\dagger}$ To whom correspondence should be addressed : akocher@Alltech.com
} 
growth and development challenges the theory of a direct antimicrobial effect of AGPs ( $\mathrm{Lu}$ et al., 2008; Niewold, 2007).

It is reasonable to expect that the increased pressure by consumer groups, supermarkets, the media and governments will see a reduction or even a ban on the use of AGPs. As a result, the industry will be faced with the challenge to find alternate tools to maintain intestinal health, intestinal integrity and the tools with which to maximise nutrient utilisation.

\section{ROLE OF MICROFLORA}

The composition of the intestinal microflora is closely related to intestinal health. A healthy ecosystem prevents the proliferation bacteria and reduces nutrients for these pathogens or even directly represses the growth of pathogens through the production of bacteriocins (Neish, 2002). Despite the fact of acknowledgement of an imbalance in the bacterial ecosystem as having significant implications on intestinal health, the understanding of the microbial ecology is still poor. Advanced microbial profiling techniques enable a snap-shot view of bacterial populations at a particular time and provide a useful tool to investigate the impact of dietary treatments on microbial profiles and the associated impact on broiler performance and health. Torok et al. (2008) linked significant changes in overall bacterial community in the ileum and ceca to the improvements in AME of barley based diets supplemented with exogenous feed enzymes, suggesting that bacterial communities have a variable capacity for energy harvest. This is supported by earlier studies, Vervaeke et al. (1979) suggesting that changes in bacterial composition reduce the breakdown of carbohydrates, resulting in increased availability of energy for the host. However, changes in available energy and improved growth performance are not always associated with an increase in 'beneficial' bacteria and a decrease in 'unwanted' bacteria. $\mathrm{Lu}$ et al. (2008) reported a significant decrease in the abundance of lactobacilli and an increase in the population of Clostridia species in ileum of chickens fed either monensin or an antimicrobial growth promoter. These authors question the commonly adopted theory that all clostridia species have the same degree of clostridial virulence associated with Clostridium perfringens, the pathogen associated with necrotic enteritis. It is speculated that the relative abundance of clostridia species may act in a competitively exclusive manner towards $C$. perfringens and also could act in a similar manner to symbiotic commensal bacteria such as $B$. thetaiotaomicron in modulating host nutrient absorption and processing (Hooper et al., 2001).

Understanding bacterial changes in the intestine are essential when evaluating specific additives. It is commonly accepted that the use of AGP increases feed efficiency, although the mechanisms are not fully understood. Numerous studies reported a reduction in the diversity of lactobacilli, in particular the abundance of Lactobacillus salivarius, which reduces the presence of bacterial bile salt hydrolase in the intestine and increase lipid digestion and fat absorption (Engberg et al., 2000; Guban et al., 2006; Kim et al., 2011). On the other hand, it is reported that the addition of pre and probiotics increases the diversity of lactobacilli in the ileum and reduces the prevalence of $C$. perfringens (Chee et al., 2010a; Kim et al., 2011). This apparent contradiction gives support to a hypothesis postulated by Lyte (2010). The author argues that the microbial community in the intestine constitutes an organ, rather than individual bacterial species, therefore bacteria in the intestine respond directly to signals from other organs as well as influencing the function of other organs in order to maintain homeostasis.

\section{MODULATING IMMUNITY AND EFFECT ON MUCIN DYNAMICS}

The homeostasis in microbial composition as well as susceptibility to disease is associated with the development of innate as well as acquired immunity. Modulation of both the innate (non-specific) and acquired (specific) immunity will increase the animal's ability to defend against a wide range of antigens. The immune system comprises of physical barriers (skin or mucosal membrane), effector molecules (e.g., lipopolysaccharide-binding proteins and mannose binding lectins) and cells (monocytes/macrophages) (Potempa and Pike, 2009). Compromising immune defences has not only significant implications on health and liveability it also significantly increases nutrient demand. Activation of the epithelial immune cells will lead to overproduction of pro- inflammatory cytokines (TNF- $\alpha$, IL-1 and IL-6). The decrease in feed intake, as well as the increased demand for specific nutrients has the biggest impact 
on nutrient availability and subsequent animal growth performance.

While the concentration of potentially inflammatory antigens is extremely high in the lumen only the direct exposure to enterocytes will result in such an immune response. The barrier between the lumen and the enterocytes is a complex layer of glycoprotein (mucins). Recently, increased focus has been placed on the impact of notional tools to manipulate the mucin layer, and subsequently reduce the impact of an overly strong immune response. The secretion of mucin by goblet cells is regulated by the family of $M U C$ genes, and gene expression can be induced by a wide range of factors (Chee, 2009). Mucin glycoproteins are essentially divided into membrane-associated mucins and secreted mucins. Secreted mucins form a protective viscous solution or unstirred water layer. Both types of mucin are rich in threonine, serine, proline, alanine, glycine and cystine (Montagne et al., 2004). Considering the rapid turn-over of mucin in the intestine, threonine is the most limiting amino acids regulating mucin dynamics. However, as Moran Jr. (2011) pointed out reduced levels of nonessential amino acids can impact on the formation of mucin.

A number of studies have shown that functional feed additives significantly influence mucin dynamics (Chee, 2009; Smirnov et al., 2005; Uni, 2007; Uni and Smirnov, 2006). These studies concluded that functional feed additives (probiotic or non-fermentable prebiotic - eg. MOS) influenced MUC2 mRNA expression thereby suggesting that mucin dynamics are influenced at transcriptional levels. It is interesting to note that Chee et al. (2010b) reported, that fluctuating dietary threonine levels had no impact on MUC2 mRNA expression, although as expected, in threonine deficient diets the thickness of the mucous layer was significantly reduced.

The composition of mucin represents potential binding sites for both commensal as well as pathogenic organism. More mature mucins are mainly acidic highly sulphated mucins which are less susceptible to bacterial proteases (Montagne et al., 2004). Reduced mucous thickness, immature composition and excessive physical erosion and dietary factors expose enterocytes to pathogens leading to the activation of pro-inflammatory effector genes (Neish, 2002). Lee et al., (2011) showed that feeding probiotic (Bacillus subtilis) to broiler chicks increases pro-inflammatory responses such as enhanced phago- cyte activity and increased nitric oxide production of macrophages. The stimulation of an innate immune response in the first 3 weeks of age enhanced the ability to control potential pathogens. In contrast, Singboottra et al. (2006) reported that inflammatory cytokine production in abdominal exudates cells from broilers exposed to yeast mannan was significantly decreased hence energy and nutrients were directed towards broiler growth rather than towards excessive immune reaction.

\section{NOVEL APPROACH - NUTRIGENOMICS AS A TOOL TO FIND FUNCTIONAL FEED ADDITIVES}

Molecular techniques to evaluate microbial communities such as Terminal Restriction Fragment Length Polymorphism (T-RFLP) combined with the use of RNA fingerprinting to evaluate expression of specific genes are useful tools to gain an understanding of the impact of dietary interventions on specific changes in microbial communities or the immune system. As shown in previous paragraphs, measuring changes of individual genes or bacterial species does make it difficult to interpret the impact of feed additives on microbial communities and the immune system. Even in a highly controlled experiment it is impossible to measure all possible changes in microbial ecology. Furthermore alteration in microbial diversity can lead to behaviour changes without any measureable immune response (Lyte et al., 1998). Considering these limitations a broader approach is needed to understand the impact of feed additives on the regulation of the network of gene expressions, all of which ultimately influence animal performance and health. The mapping of the genome of chickens have allowed for the development of microarray as a tool to research the effect of specific nutrients on functional gene network and regulatory pathways (International Chicken Genome Sequencing Consortium, 2004; International Chicken Polymorphism Map Consortium, 2004). Many changes seen at gene expression level within a specific organ or tissue are much more subtle than can be measured in animal growth and physiology. Nutrigenomics is the science which studies the relationship between nutrition, and the specific effects of nutritional additives on the response of genes. However, it is important to note that it is not always possible to directly correlate an 
increased present of mRNA tissue samples as a reflection of higher gene expression, with phenotypic or protein changes in tissue (Moody, 2001).

Early steps in this direction have been presented by Xiao et al. (2010). This study looked at the molecular mechanisms comparison of two fractions from the yeast cell wall. The study found that yeast cell wall fractions alter the expression of over 1,500 genes in the jejunum of broilers compared to an unsupplemented control. In particular it was shown that genes related to pathogen defences were significantly induced whereas the expression of genes related to the inflammatory response were decreased. Although it is still in its infancy, the use of pathway analysis will provide a much greater insight into the effect of feed additives to maintain intestinal health and integrity.

\section{CONCLUSIONS}

One of the biggest challenges facing the poultry industry is the minimisation of waste of valuable nutrients as a result of damage to the intestine. Advances in the understanding of dietary interventions on disease resistance and production efficiencies as a result of developments in the field of Nutrigenomics will give scientists and producers a more precise tool to differentiate and identify strategies to maximize animal performance.

\section{LITERATURE CITED}

Chee SH 2009 Functional interactions of mannooligosaccharides with dietary threonine on chicken gastrointestinal tract. $\mathrm{PhD}$, University of New England.

Chee SH, Iji PA, Choct M, Mikkelsen LL, Kocher A 2010a Characterisation and response of intestinal microflora and mucins to manno-oligosaccharide and antibiotic supplementation in broiler chickens. Br Poult Sci 51:368-380.

Chee SH, Iji PA, Choct M, Mikkelsen LL, Kocher A 2010b

Functional interactions of manno-oligosaccharides with dietary threonine in chicken gastrointestinal tract. I. Growth performance and mucin dynamics. Br Poult Sci 51:658666.

Engberg RM, Hedemann MS, Leser TD, Jensen BB 2000
Effect of zinc bacitracin and salinomycin on intestinal microflora and performance of broilers. Poultry Sci 79:13111319.

Guban J, Korver DR, Allison GE, Tannock GW 2006 Relationship of dietary antimicrobial drug administration with broiler performance, decreased population levels of Lactobacillus salivarius, and reduced bile salt deconjugation in the ileum of broiler chickens. Poultry Sci 85:2186-2194.

Hooper LV, Wong MH, Thelin A, Nansson L, Falk PG, Gordon JI 2001 Molecular analysis of host-microbial relationship in the intestine. Science 291:881-884.

International Chicken Genome Sequencing Consortium 2004 Sequence and comparative analysis of the chicken genome provide unique perspectives on vertebrate evolution. Nature 432:695-716.

International Chicken Polymorphism Map Consortium 2004 A genetic variation map for chicken with 2.8 million singlenucleotide polymorphisms. Nature 432:717-722.

Kim GB, Seo YM, Kim CH, Paik IK 2011 Effect of dietary prebiotic supplementation on the performance, intestinal microflora, and immune response of broilers. Poultry Sci 90: 75-82.

Lee KW, Li G, Lillehoj HS, Lee SH, Jang SI, Babu US, Lillehoj EP, Neumann AP, Siragusa GR 2011 Bacillus subtilis-based direct-fed microbials augment macrophage function in broiler chickens. Res Vet Sci 91:e87-91.

Lu J, Hofacre C, Smith F, Lee MD 2008 Effects of feed additives on the development on the ileal bacterial community of the broiler chicken. Animal 2:669-676.

Lyte M 2010 The microbial organ in the gut as a driver of homeostasis and disease. Med Hypotheses 74:634-638.

Lyte M, Varcoe JJ, Bailey MT 1998 Anxiogenic effect of subclinical bacterial infection in mice in the absence of overt immune activation. Physiol Behav 65:63-68.

Montagne L, Cavaney FS, Hampson DJ, Lalles JP, Pluske JR 2004 Effect of diet on mucin kinetics and composition: nutrition and health implications. Nutr Rev 62:105-114.

Moody DE 2001 Genomics techniques: An overview of methods for the study of gene expression. J Anim Sci 79: E128-E135.

Moran Jr. ET 2011 Crude protein requirement and maintenance of the intestine. In 'Australian Poultry Science Sym- 
posium'. Sydney, Australia. (Ed. TA Scott) pp. 1-8. (University of Sydney).

Neish AS 2002 The gut microflora and intestinal epithelial cells: A continuing dialogue. Microbes Infect 4:309-317.

Niewold TA 2007 The nonantibiotic anti-inflammatory effect of antimicrobial growth promoters, the real mode of action? A Hypothesis. Poultry Sci 86:605-609.

Potempa J, Pike RN 2009 Corruption of innate immunity by bacterial proteases. J Innate Immun 1:70-87.

Roppa L 2007 Protein demand drives poultry production. World Poultry 23:27-28.

Singboottra P, Edens F, Kocher A 2006 Mannan induced changes in cytokine expression and growth of enteropathogenic E. coli-challenged broilers. In 'Gut Microbiology 2006: 5th Biennial Meeting Research to Improve Health, Immune Response and Nutrition'. Aberdeen p. S74. (RRI-INRA).

Smirnov A, Perez Vendrell AM, Amit-Romach E, Sklan D, Uni Z 2005 Mucin dynamics and microbial populations in chicken small intestine are changed by dietary probiotic and antibiotic growth promoter supplementation. J Nutr 135:187-192.

Speedy AW 2003 Global production and consumption of animal source foods. J Nutr 133:4048S-4053S.

Torok VA, Ophel-Keller K, Loo M, Hughes RJ 2008 Application of methods for identifying broiler chicken gut bacterial species linked with increased energy metabolism. Appl
Environ Microbiol 74:783-791.

Uni Z 2007 Physiological dynamics at the intestinal brush border. In 'Biotechnology in the Feed Industry: Proceedings of Alltech's 23th Annual Symposium'. (Eds TP Lyons, KA Jacques, JM Hower) pp. 131-138 (Nottingham Press: Nottingham).

Uni Z, Smirnov A 2006 Modulating mucin dynamics using functional carbohydrates. In 'Gut microbiology: Research to Improve Health, Immune Response and Nutrition. Fifth RRIINRA Joint Symposium'. Aberdeen, UK.

United Nation 2011 The World at Six Billion.

van der Klis JD 2010 Challenges to European poultry production. In 'Australian Poultry Science Symposium'. Sydney pp. 17-21 (University of Sydney).

Vervaeke IJ, Decuypere JA, Dierick NA, Henderickx HK 1979 Quantitative in vitro evaluation of the energy metabolism influenced by virginiamycin and spiramycin used as growth promoters in pig nutrition. J Anim Sci 49:846-856.

Xiao R, Power RF, Mallonee D, Crowdus C, Ao T, Pierce JL, Dawson KA 2010 Transcriptional signatures associated with biological functions of Bio-Mos ${ }^{\circledR}$ and Actigen $^{\mathrm{TM}}$ in broilers. In 'Biotechnology in the Feed Industry: Proceedings of Alltech's 26th Annual Symposium'. Lexington, KY. (Eds TP Lyons, KA Jacques, JM Hower).

(submitted: 2011. 11. 18, revised: 2011. 12. 20, accepted: 2011. 12. 28) 